\title{
Paepalanthus magistrae (Eriocaulaceae), a remarkable new species in honor of Ana Maria Giulietti-Harley
}

\author{
Paulo Takeo Sano ${ }^{1}$, Fabiane Nepomuceno Costa ${ }^{2}$, Marcelo Trovó ${ }^{3} \&$ Lívia Echternacht ${ }^{4}$
}

\begin{abstract}
We describe and illustrate Paepalanthus magistrae, a remarkable new species of Eriocaulaceae from Northeastern Brazil. The species is placed into Paepalanthus subsect. Dichocladus by the presence of trimerous flower, elongated dichotomous branched stem, and small rigid leaves. Within the section it may be easily distinguished by its leaves with glabrescent adaxial surface and lanose abaxial surface. As the species is narrowly distributed and known from a few populations, it is considered endangered.
\end{abstract}

Key words: Caatinga, Paepalanthoideae, Serra das Confusões National Park, taxonomy.

\section{Introduction}

Paepalanthus Mart. is the richest genus of Eriocaulaceae in Brazil with ca. 340 species, $95 \%$ of which restricted to the country, with a high number of micro-endemic species (Giulietti et al. 2010; Forzza et al. 2010; Sano et al. 2014). New species are frequently described, especially for poorly sampled areas (e.g. Giulietti \& Miranda 2009; Trovó \& Sano 2009, 2011; Trovó et al. 2011, 2012, 2013a, 2013b; Echternacht \& Trovó in press). The Espinhaço Range in Southeastern Brazil is the center of diversity of the genus, which is also quite diverse on the Guiana Shield and, in a lower degree, on Central Brazil and on the Atlantic Forest domain. Piaui state, in northeastern Brazil, is particularly poor in Paepalanthus, with only four species acknowledged in the Brazilian checklist (Sano et al. 2014). The Serra das Confusões National Park is located at the south of the state, mostly within the Caatinga domain. There, sandstone outcrops, especially on the slopes of the Mountains, are formed by quartzose soils, the typical environment for Eriocaulaceae.

Paepalanthus differs from the remaining genera of Eriocaulaceae by its isostemonous flowers, the pistillate with free petals, and gynoecium with stigmatic and nectariferous branches inserted at the same point (Koernicke 1863; Giulietti et al. 2012). The genus was revealed polyphyletic (Andrade et al. 2010; Giulietti et al. 2012; Trovó et al. 2013c), as well as several of its 28 supra-specific ranks defined by Ruhland (1903). Paepalanthus subsect. Dichocladus Ruhland includes plants with trimerous flowers, elongated and dichotomously branched stems, and small, rigid leaves. The subsection includes a group of species from the Espinhaço Range that form a clade, and a disjunctive group from the Guiana Shield, which were not yet included in phylogenetic analyses (Trovó et al. 2013c). The habit enables an easy association of species to $P$. subsect. Dichocladus, but the monophyly of the whole taxon is still to be tested. Recent collections from the Serra das Confusões revealed a new, very distinctive species of $P$. subsect. Dichocladus that is described herein.

\section{Taxonomic treatment}

Paepalanthus magistrae Sano, F.N. Costa, Trovó \& Echtern. sp. nov.

Type: Brazil. Piauí: Caracol. "Parque Nacional da Serra das Confusões, afloramento rochoso na localidade Moquem, próximo da gruta do Enoque. $9^{\circ} 08^{\prime} 11^{\prime \prime} \mathrm{S}, 43^{\circ} 33^{\prime} 27^{\prime \prime} \mathrm{W}$. Elev. 500m". $22 \mathrm{Feb}$ 2013, Martinelli, G. et. al. 18122 (holotype: RB, isotype: B).

Fig. 1,2.

Diagnosis: Paepalanthus magistrae differs from all the other species of $P$. subsect. Dichocladus

\footnotetext{
${ }^{1}$ Universidade de São Paulo, Depto. Botânica, Inst. Biociências, R. do Matão 277, 05508-900, São Paulo, SP, Brasil. ptsano@usp.br

${ }^{2}$ Universidade Federal dos Vales do Jequitinhonha e Mucuri, Depto. Ciências Biológicas, Campus JK, Rodovia BR-367, 39100-000, Diamantina, MG, Brasil. fncosta@hotmail.com

${ }^{3}$ Universidade Federal do Rio de Janeiro, Depto. Botânica, Inst. Biologia, CCS, Bloco A1, Cidade Universitária, 21941-590, Rio de Janeiro, RJ, Brasil. martrovo@gmail.com

${ }^{4}$ Universidade Federal de Uberlândia, Inst. Biologia, Campus Umuarama, R. Ceará s/n, 38400-902, Uberlândia, MG, Brasil. livia.echter@inbio.ufu.br
} 
by its leaves with glabrescent adaxial surface and lanose abaxial surface. It is morphologically differentiated from Paepalanthus bonsai Trovó \& Sano, the most closely related species, also by its leaves with round apex, spathe tip acute, longer scapes, spherical capitula, and involucral bracts completely glabrous.
Perennial herbs forming small, dense cushions in rocky crevices, $4.5-11 \mathrm{~cm}$ tall, stem elongate, 4-7.5 cm long, branched, covered by leaves and marcescent leaf sheaths. Leaves disposed along the stem, chartaceous, deciduous, falciform, 5-7 × 0.5-0.1 mm, adaxial surface glabrescent, abaxial surface lanose, apex round,
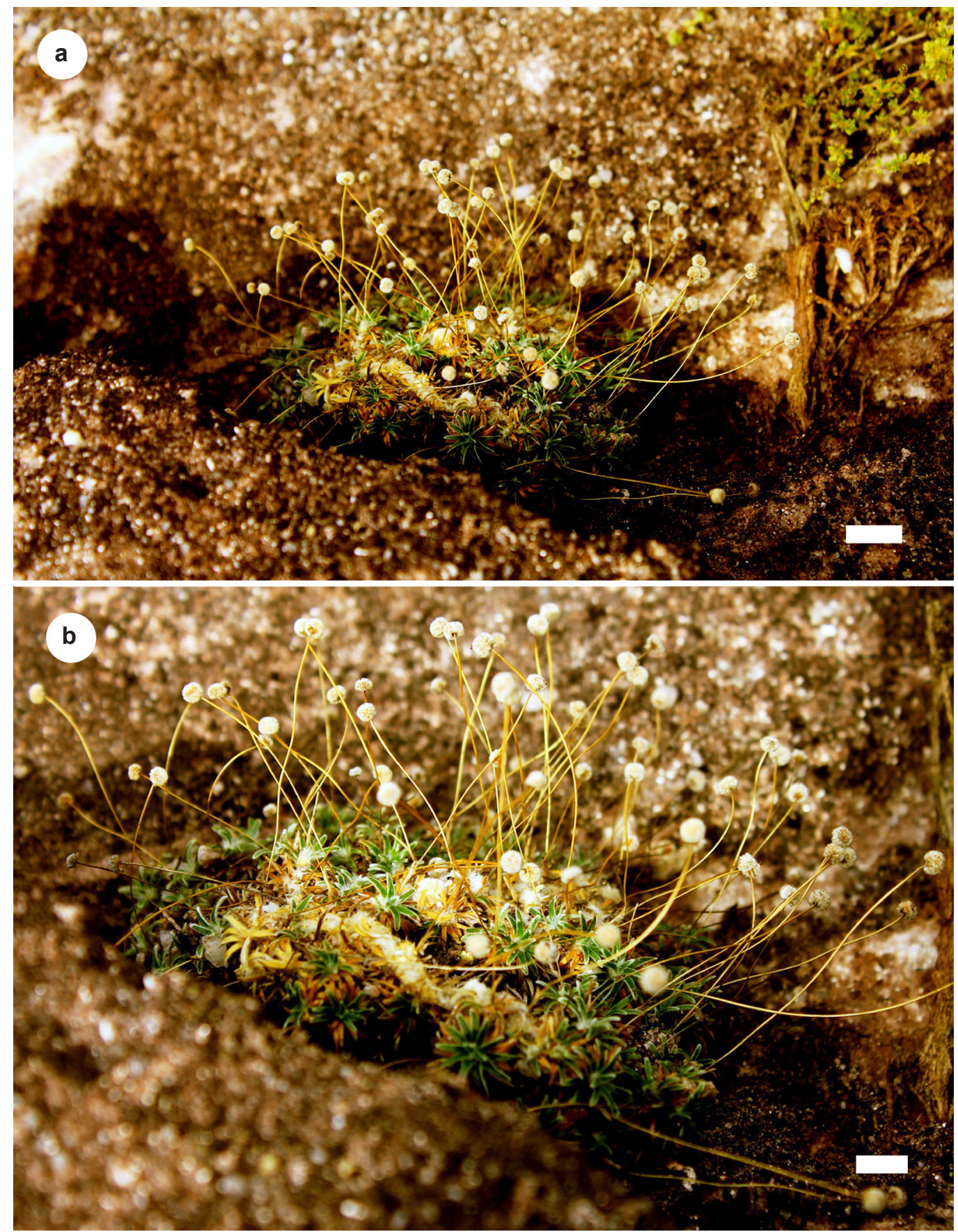

Figure 1 - Paepalanthus magistrae - a. habit; b. habit detail. Scale bars - a. $2.5 \mathrm{~cm}$; b. $1 \mathrm{~cm}$. (photos from CNCFlora) 


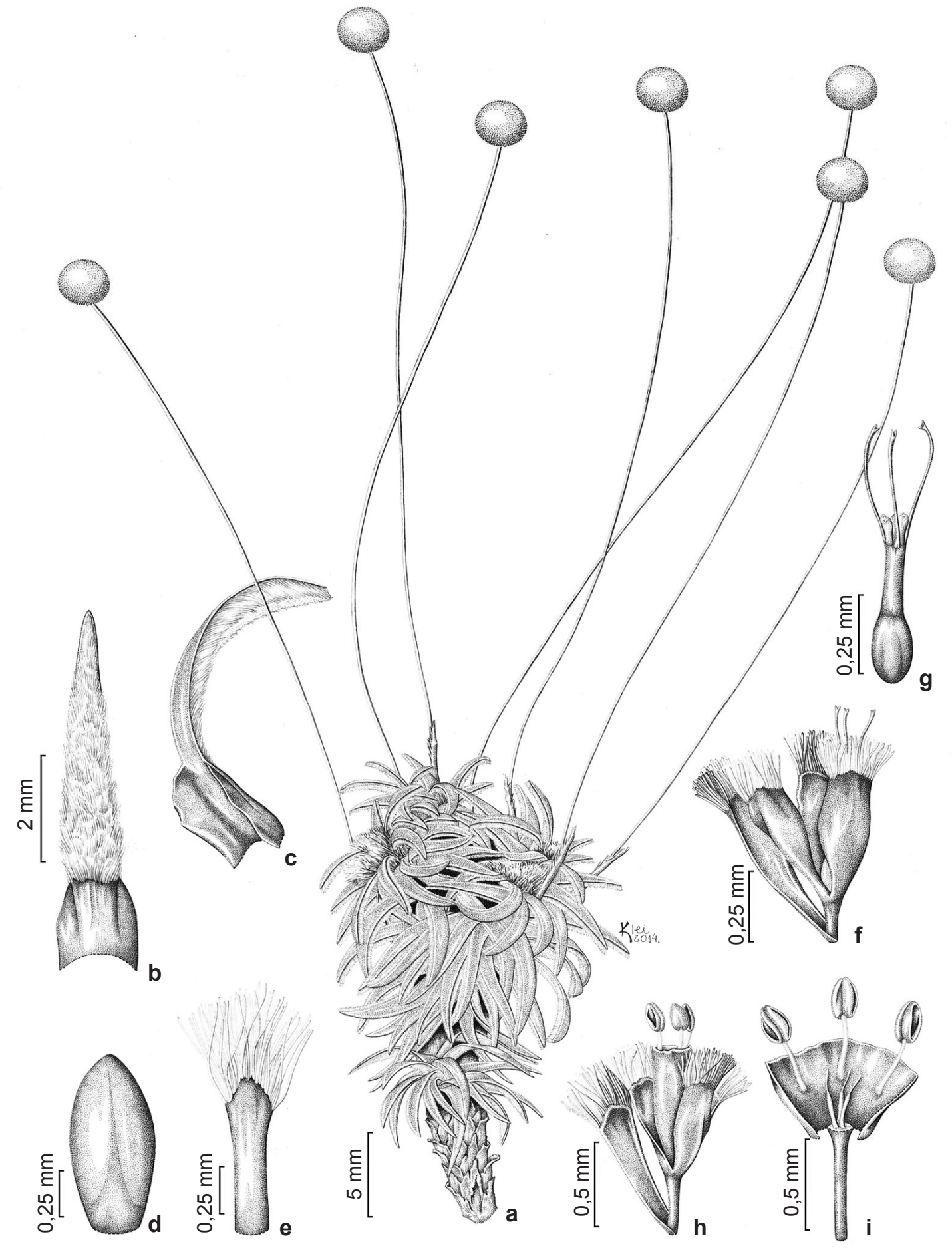

Figure 2 - Paepalanthus magistrae - a. branch detail; b. leaf abaxial surface; c. leaf adaxial surface; d. involucral bract abaxial surface; e. floral bract abaxial surface; f. pistillate flower; g. gynoecium; h. staminate flower; i. staminate flower with sepals removed and opened corolla (Drawings from the holotype by Klei Sousa). 
base dilated, and margins glabrous. Spathes 0.5-1 $\mathrm{cm}$ long, lanose to glabrescent, tip acute. Scapes 1 to 3 per branch, 2-6.5 cm long, glabrous; capitula 2-4 mm diam., spherical; involucral bracts light brown with a dark stripe in the middle, arranged in 2 series, deltoid, ca. $2 \mathrm{~mm}$ long, glabrous on both surfaces, apex obtuse, margin glabrous; receptacle hemispheric, pubescent. Flowers 3-merous, ca. 50 per capitulum, $4 \times$ more staminates than pistillates; floral bracts narrowly oblong, ca. $2 \mathrm{~mm}$ long, glabrous, apex acute, ciliate toward the apex. Staminate flowers ca. $3 \mathrm{~mm}$ long; pedicel ca. 0.5 $\mathrm{mm}$ long, with long trichomes; sepals oblong, ca. $1.5 \mathrm{~mm}$, glabrous, apex acute, ciliate toward the apex; corolla tubular, membranaceous, hyaline, glabrous, ca. $2.5 \mathrm{~mm}$ long, truncated; stamens ca. $2.5 \mathrm{~mm}$; pistillodes 3, long papillose. Pistillate flowers ca. $1.5 \mathrm{~mm}$, sessile; sepals oblong, ca. 2 mm long, glabrous, apex acute, ciliate toward the apex; petals obovate, ca. $1.5 \mathrm{~mm}$ long, glabrous, apex obtuse, ciliate toward the apex; gynoecium ca. $1.5 \mathrm{~mm}$ long, stigmatic branches bifid, twice the length of the small nectariferous branches. Fruit a loculicidal capsule.

Etymology: The epithet magistrae belonging to the master - is a tribute to Prof. Ana Maria Giulietti-Harley, who has guided three generations of specialists in Eriocaulaceae. As in this species, she has her roots in the Caatinga. To her we offer our gratitude and our acknowledgement.

Habitat, Distribution, and Conservation: Paepalanthus magistrae is known from a few populations growing on rock crevices in mountains of Serra das Confusões, Piauí (Fig. 1). The species occurs within the Capivara National Park and is considered endangered according to criteria B1a and B2a of IUCN (2011). Additional populations may be found in the vicinities, as the area is poorly sampled.

Notes: Paepalanthus magistrae is placed into Paepalanthus subsect. Dichocladus due to its trimerous flowers, elongated dichotomous branched stem, and small rigid leaves. Within the section, it is similar to P. bonsai and P. glaziovii Ruhland by possessing bifid stigmatic branches. Differs from $P$. bonsai, the most similar species, by its leaves with abaxial surface lanose (vs. glabrescent), leaf apex rounded (vs. acute), spathe tip acute (vs. truncate), longer scapes, spherical capitula (vs. urceolate), involucral bracts glabrous (vs. pilose in the abaxial surface and margin), and small flower differences. Despite the morphological differences, $P$. magistrae is allopatric distributed, as both $P$. bonsai and $P$. glaziovii are restricted to the Diamantina Plateau in Minas Gerais state.

Paratypes: BRAZIL. PIAUÍ: São Raimundo Nonato, "Serra da Capivara", 10.VII.1984, Emperaire, L. 2583 (RB). Guaribas, "Serra das Confusões", 1-15.X.2000, Percequillo, A.R. et al. (SPF 144286).

\section{Acknowledgments}

Financial support: M.T., Alexander von Humboldt Foundation, UFRJ (ALV 2013) and FAPERJ (E-26/112.476 - INST; E-26/110.031/2011, E-26/111.392/2012 - BIOTA); P.T.S., CNPq (proc. 308300/2012-2); L.E., PROPP-UFU (edital 06/2013).

\section{References}

Andrade, M.J.; Giulietti, A.M.; Rapini, A.; Queiroz, L.P.; Conceição, A.D.S.; Almeida, P.R.M. \& van den Berg, C. 2010. A comprehensive phylogenetic analysis of Eriocaulaceae: Evidence from nuclear (ITS) and plastid ( $p s b A$-trnH and $t r n L-F$ ) DNA sequences. Taxon 59: 379-388.

Echternacht, L.A. \& Trovó, M. 2015. Paepalanthus serpens, a new microendemic species of Eriocaulaceae from the Espinhaço Range, Minas Gerais, Brazil. PhytoKeys 48: 43-49.

Forzza, R.C.; Baumgratz, J.F.A.; Bicudo, C.E.; Carvalho Jr., A.A.; Costa, A.; Costa, D.P.; Hopkins, M.; Leitman, P.M.; Lohmann, L.G.; Maia, L.C.; Martinelli, G.; Menezes, M.; Morim, M.P.; Nadruz Coelho, M.A.; Peixoto, A.L.; Pirani, J.R.; Prado, J.; Queiroz, L.P.; Souza, V.C.; Stehmann, J.R.; Sylvestre, L.S.; Walter, B.M.T. \& Zappi, D. 2010. Catálogo de Plantas e Fungos do Brasil. Vol. 1. Andrea Jakobsson Estúdio, Instituto de Pesquisas Jardim Botânico do Rio de Janeiro, Rio de Janeiro. 871p.

Giulietti, A.M. \& Miranda, E.B. 2009. Five new taxa in Eriocaulaceae from the Chapada Diamantina of Bahia, Brazil. Kew Bulletin 64: 525-536.

Giulietti, A.M.; Sano, P.T.; Costa, F.N.; Parra, L.R.; Echternacht, L.; Tissot-Squali, M.L.; Trovó, M.; Watanabe, M.T.C.; Freitas, M.P. \& Hensold, N. 2010. Eriocaulaceae. In: Forzza, R.C. et al. Catálogo de Plantas e Fungos do Brasil. Vol. 2. Andrea Jakobsson Estúdio \& Instituto de Pesquisas Jardim Botânico do Rio de Janeiro, Rio de Janeiro. Pp.938-958.

Giulietti, A.M.; Andrade, M.J.G.; Scatena, V.L.; Trovó, M.; Coan, A.I.; Sano, P.T.; Santos, F.A.R.; Borges, R.L.B. de \& van den Berg, C. 2012. Molecular phylogeny, morphology and their implications for the taxonomy of Eriocaulaceae. Rodriguésia 63: 1-19.

IUCN Standards Petitions Sub-committee. 2011. Guidelines for using the IUCN Red List Categories and Criteria, Version 9.0. Available at $<$ http:// www.iucn.org>. Access on 10 April 2014. 
Koernicke, F. 1863. Eriocaulaceae. In: Martius, K. F. P. \& Eichler, A. W. Flora Brasiliensis. Typographia Regia, Munchen,. Vol. 3, pp. 271-508.

Ruhland, W. 1903. Eriocaulaceae. In: Engler, A. Das Pflanzenreich. Vol. 4.30. Regni vegetabilis conspectus. Engelmann, Leipzig. Pp. 1-294.

Sano, P.T., Giulietti, A.M., Costa, F.N., Parra, L.R., Echternacht, L., Tissot-Squali, M.L., Trovó, M., Watanabe, M.T.C., Freitas, M.P. \& Hensold, N. 2014. Eriocaulaceae. In: Lista de Espécies da Flora do Brasil. Jardim Botânico do Rio de Janeiro. Available at <http://reflora.jbrj.gov.br/jabot/floradobrasil/ FB110>. Access on 24 August 2014.

Trovó, M. \& Sano, P.T. 2009. Paepalanthus bonsai, a New Species of Eriocaulaceae from Minas. Novon 19: 412-415.

Trovó, M. \& Sano, P.T. 2011. Five New Narrowly Distributed Species of Paepalanthus Section Diphyomene (Eriocaulaceae) from Central Brazil. Systematic Botany 36: 1-11.
Trovó, M., Echternacht, L. \& Sano, P.T. 2011. Paepalanthus hirtellus, a new species of Paepalanthus subsection Actinocephaloides (Eriocaulaceae) from Minas Gerais, Brazil. Phytotaxa 15: 26-32.

Trovó, M., Echternacht, L. \& Sano, P.T. 2012. Paepalanthus sphaeroides, a new species of Eriocaulaceae from the Atlantic Forest, Brazil. Blumea 57: 105-108.

Trovó, M., Echternacht, L. \& Sano, P.T. 2013a. Three New Species of Paepalanthus sect. Diphyomene (Eriocaulaceae) from Minas Gerais, Brazil. Novon: 22: 325-331.

Trovó, M., Echternacht, L., Sano, P.T. \& Costa, F.N. 2013b. Paepalanthus aleurophyllus, a new species of Eriocaulaceae from Minas Gerais, Brazil. Blumea 58: 77-79.

Trovó, M., Andrade, M.J.G. de, Sano, P.T., Ribeiro, P.L. \& van den Berg, C. 2013c. Molecular phylogenetics and biogeography of Neotropical Paepalanthoideae with emphasis on Brazilian Paepalanthus (Eriocaulaceae). Botanical Journal of the Linnean Society 171: 225-243. 
